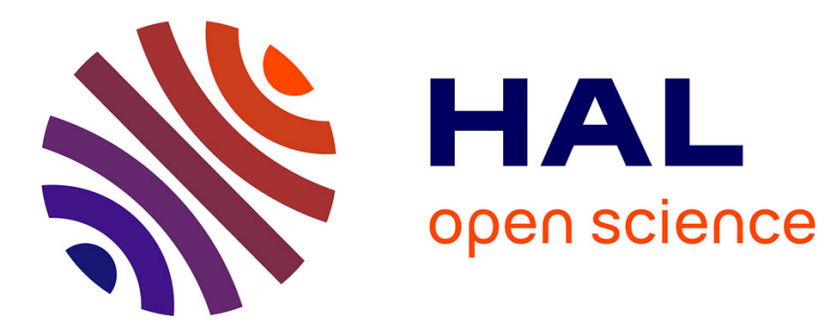

\title{
Joint contrast optimization and object segmentation in active polarimetric images
}

Guillaume Anna, Nicolas Bertaux, Frédéric Galland, François Goudail, Daniel Dolfi

\section{- To cite this version:}

Guillaume Anna, Nicolas Bertaux, Frédéric Galland, François Goudail, Daniel Dolfi. Joint contrast optimization and object segmentation in active polarimetric images. Optics Letters, 2012, 37 (16), pp.3321-3323. hal-00747209

\section{HAL Id: hal-00747209 \\ https://hal-iogs.archives-ouvertes.fr/hal-00747209}

Submitted on 30 Oct 2012

HAL is a multi-disciplinary open access archive for the deposit and dissemination of scientific research documents, whether they are published or not. The documents may come from teaching and research institutions in France or abroad, or from public or private research centers.
L'archive ouverte pluridisciplinaire HAL, est destinée au dépôt et à la diffusion de documents scientifiques de niveau recherche, publiés ou non, émanant des établissements d'enseignement et de recherche français ou étrangers, des laboratoires publics ou privés. 


\title{
Joint contrast optimization and object segmentation in active polarimetric images
}

\author{
Guillaume Anna, ${ }^{1}$ Nicolas Bertaux, ${ }^{2}$ Frédéric Galland, ${ }^{2}$ François Goudail, ${ }^{1, *}$ and Daniel Dolfi ${ }^{3}$ \\ ${ }^{1}$ Laboratoire Charles Fabry, UMR 8501, Institut d'Optique, CNRS, Université Paris Sud 11, 91127 Palaiseau, France \\ ${ }^{2}$ Institut Fresnel, Aix-Marseille Université, Ecole Centrale Marseille, CNRS, Campus de St. Jérôme, 13013 Marseille, France \\ ${ }^{3}$ Thales Research and Technology—France, RD128, 91767 Palaiseau Cedex, France \\ *Corresponding author: francois.goudail@institutoptique.fr
}

Received May 21, 2012; revised June 22, 2012; accepted June 28, 2012;

posted June 29, 2012 (Doc. ID 168678); published August 6, 2012

\begin{abstract}
We present a method for automatic target detection based on the iterative interplay between an active polarimetric imager with adaptive capabilities and a snake-based image segmentation algorithm. It successfully addresses the difficult situations where the target and the background differ only by their polarimetric properties. This method illustrates the benefits of integrating digital processing algorithms at the heart of the image acquisition process rather than using them only for postprocessing. (ㄷ 2012 Optical Society of America

OCIS codes: $110.5405,100.0100$.
\end{abstract}

Active polarimetric imaging is a powerful tool for revealing contrasts that do not appear in standard intensity images and has been proven useful in such domains as remote sensing or biomedical imaging [1,2]. Extensive research has been conducted to maximize the contrast between an object of interest and a background in target detection applications [3-6]. Most of these studies assumed that the polarimetric properties (i.e., the Mueller matrices) of the objects in the scene were known beforehand. This is obviously a limitation to their practical use. The purpose of the present Letter is to propose a solution to this issue that consists in performing iteratively image segmentation and contrast optimization. It is based on the iterative operation of an active polarimetric imager whose illumination and analysis polarization states can be anywhere on the Poincaré sphere and of a fast and unsupervised image segmentation algorithm. The benefits of this approach will be demonstrated on real-world images in difficult situations where target and background differ only by polarimetric properties.

Let us consider an active polarimetric imaging system that illuminates the scene with light whose polarization state is defined by a Stokes vector $\mathbf{S}$ and is produced by a polarization state generator (PSG) [7]. The polarimetric properties of a region of the scene corresponding to a pixel in the image is characterized by its Mueller matrix $M$. The Stokes vector of the light scattered by this region is $\mathbf{S}^{\prime}=M \mathbf{S}$. It is analyzed by a polarization state analyzer (PSA), which is a generalized polarizer allowing selecting photons characterized by their polarization state $\mathbf{T}$. The number of photoelectrons measured at a pixel of the sensor is

$$
i=\frac{\eta I_{0}}{2} \mathbf{T}^{T} M \mathbf{S},
$$

where the superscript $T$ denotes matrix transposition. In this equation, $\mathbf{S}$ and $\mathbf{T}$ are unit intensity, purely polarized Stokes vectors, $I_{0}$ is a number of photons, and $\eta$ is the conversion efficiency between photons and electrons. By changing the PSG and PSA states, it is possible to optimize the contrast between a target of interest and the background in the image $[\underline{3}, \underline{6}]$. However, this optimization assumes that the Mueller matrices of the regions of interest are known, and the question remains of what can be done if these matrices are unknown.

A first solution would be to measure the full Mueller image of the scene, which consists in acquiring 16 intensity images with 16 different couples of PSG/PSA states. An image segmentation algorithm would then be used to automatically extract the shape of the target. However, acquisition of 16 images with sufficient signal-to-noise ratio (SNR) may require a long time, which can be a problem in the presence of rapidly evolving scenes. Furthermore, automatic segmentation algorithms on high-dimensional, noisy data with strong inhomogeneities [6] are prone to errors, such as being stuck in local maxima of the segmentation criterion.

To solve these issues, we propose an iterative method based on the acquisition of single images with optimized contrast [7]. It is illustrated in Fig. 1. The first step consists in acquiring the full Mueller image in $t_{0}$ seconds. The integration time of each images is thus about $t_{0} / 16$, and they consequently have a low SNR. We then use a fast and unsupervised segmentation algorithm adapted to such noisy 16-dimensional (16D) images (described below) that gives a first estimation of the target shape. Because of low SNR and inhomogeneities in some components, this shape estimation is not perfect. However, from this first segmentation, it is possible to estimate the polarimetric properties of the pixels inside and outside the segmented region (object and background). One estimates the average Mueller matrix

$$
\bar{M}_{u}=\frac{1}{N_{\Omega_{u}}} \sum_{k \in \Omega_{u}} M_{u}^{k}
$$

with $u=\{$ in, out $\}$ corresponding to pixels inside or outside the segmentation boundaries, $\Omega_{u}$ the set containing the $N_{\Omega_{u}}$ pixels in the region $u$, and $M_{u}^{k}$ the Mueller matrix of the pixel $k$ in the region $u$. The spatial fluctuations are characterized by the covariance matrix given by

$$
G_{u}=\frac{1}{N_{\Omega_{u}}} \sum_{k \in \Omega_{u}}\left[\mathbf{V}_{M_{u}^{k}}-\mathbf{V}_{\bar{M}_{u}}\right]\left[\mathbf{V}_{M_{u}^{k}}-\mathbf{V}_{\bar{M}_{u}}\right]^{T},
$$

with $\mathbf{V}_{M}$ the vectorized Mueller matrix $M$. 

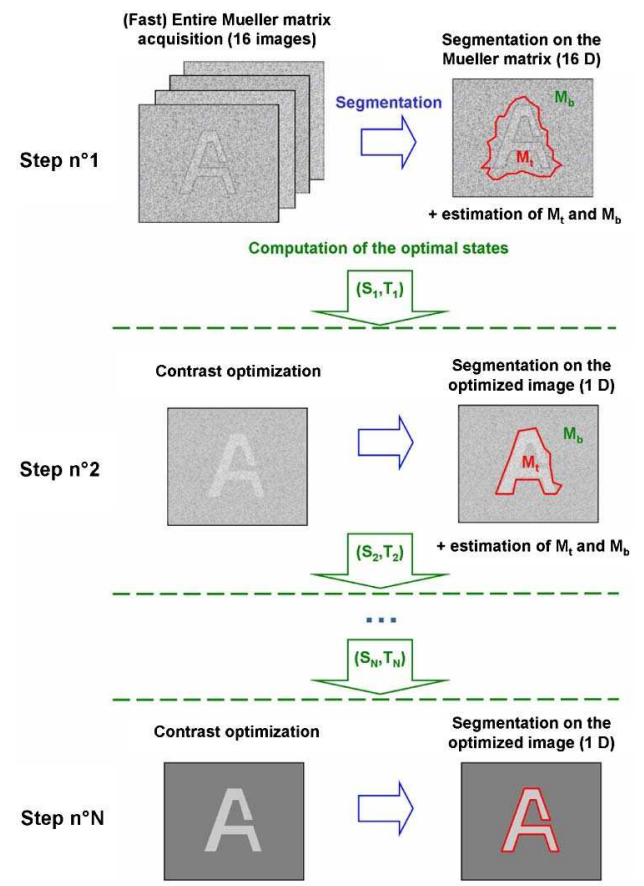

Fig. 1. (Color online) Different steps to detect and recognize a target from a background.

Using these estimates, the PSG and PSA states $\mathbf{S}_{1}$ and $\mathbf{T}_{1}$ that maximize the contrast between the two regions are determined by optimizing the Fisher ratio []]:

$$
\mathcal{F}=\frac{\left[\bar{x}_{\text {in }}-\bar{x}_{\text {out }}\right]^{2}}{\operatorname{var}\left(x_{\text {in }}\right)+\operatorname{var}\left(x_{\text {out }}\right)},
$$

with $\quad \bar{x}_{u}=\mathbf{T}^{T} \bar{M}_{u} \mathbf{S}$ and $\operatorname{var}\left(x_{u}\right)=[\mathbf{T} \otimes \mathbf{S}]^{T} G_{u}[\mathbf{T} \otimes \mathbf{S}]$ (the symbol $\otimes$ denotes the Kronecker product). These optimal states are implemented on the imaging system to acquire a single image with an integration time equal to $t_{0}$ and thus a better SNR. This image is then segmented in order to refine the shape of the target, which allows one to obtain a better estimation of the polarimetric properties of the target and the background $\left(M_{u}, G_{u}\right.$, $u \in\{$ in, out $\})$ and thus a better estimation of the optimal PSG/PSA states $\mathbf{S}_{2}$ and $\mathbf{T}_{2}$. These new states can be implemented on the imaging system to acquire a new image, which is segmented to further refine the PSG/PSA estimation. This process of acquisition/segmentation/contrast optimization can be iterated until the contrast between the target and the background is sufficient. The number of iterations will depend on the complexity of the scene and on the difference of polarimetric properties between the target and the background. However, since all the steps but the first one are based on single image acquisitions, it is much more robust to object movements in the scene.

One of the key elements of this method is the segmentation algorithm. First, it should be fast and not require any intervention from the user. It should also be adapted to both one-dimensional (1D) and 16D noisy intensity images whose fluctuations may not be correctly modeled with standard probabilities laws, due notably to illumination inhomogeneities and textures in the image. We thus used the polygonal active contour (snake) proposed in
[8,9], initially developed for $1 \mathrm{D}$ images and that has been generalized in this Letter to multidimensional images. This algorithm relies on a minimum description length (MDL) criterion, based on a nonparametric description of the gray level fluctuations that are modeled with $K$-bin histograms. Both the number and location of the nodes of the polygonal contour used to separate the object from the background are estimated iteratively via the optimization of the MDL criterion, which does not contain any parameter to be tuned by the user.

The most time-consuming step in this algorithm is the calculus of the $K$ statistics needed to update the height of the $K$ bins of the histogram after each deformation of the contour [9]. Fast computation of these statistics are obtained with summing $K$ precomputed images along the contour of the object, and a number of bins $K$ between 8 and 16 usually yields a good trade-off between computation time and discrimination capability. The computation time of this operation can be significantly reduced by vectorizing it with the streaming single instruction multiple data (SIMD) extensions (SSEs). As described in [9], for images with a pixel number $N \leq 32767$, it allows computing $S=8$ statistics simultaneously with only one SSE summation. Assuming that the gray level values of the 16 Mueller matrix components are independent, the generalization of the $1 \mathrm{D}$ MDL criterion to $16 \mathrm{D}$ is straightforward [8], the data adequacy term in the MDL criterion being simply the sum of the data adequacy terms for each of the 16 components. It is thus necessary to calculate $16 K$ statistics, which implies $k$ SSE summations, where $k$ is the smallest integer not less than $(16 K) / S$. Since in the considered images $N \leq 32767, S=8$, and thus only $k=2 K$ SSE summations are necessary, allowing to keep a reduced computation time even when dealing with $16 \mathrm{D}$ intensity images. For example, a $152 \times 162$ pixel image (see Fig. 3 below) is segmented with $K=8$ in $4 \mathrm{~ms}$ for $1 \mathrm{D}$ images and less than $20 \mathrm{~ms}$ for $16 \mathrm{D}$ images on a $2.5 \mathrm{GHz}$ processor laptop.

Let us now apply this method on a real-world image. The scheme of the scene is represented in Fig. 2(a). The target is composed of metallic plates and the background of white paper. Both are placed behind a piece of diffusing paper and a scattering birefringent medium; they cannot be discriminated on a standard intensity image [see Fig. 2(b)]. We first acquire full Mueller data with a short integration time of $t_{0} / 16=150 \mu \mathrm{s}$ for each of the 16 images, and we apply the $16 \mathrm{D}$ segmentation algorithm to extract a first estimated shape of the target (see Fig. $\underline{3}$, Step 1). Even if the contour encloses the target, its shape is not well defined due to the presence of noise and spatial fluctuations of the polarimetric properties in the

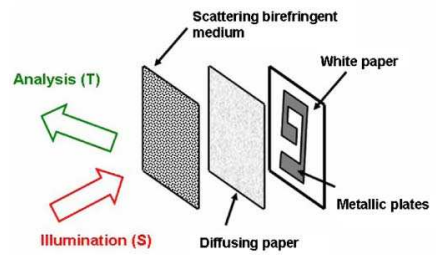

(a)

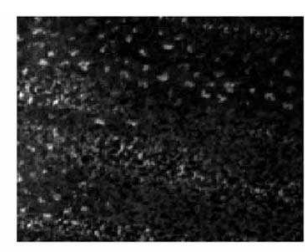

(b)
Fig. 2. (Color online) (a) Scheme of the scene. (b) Intensity image. 


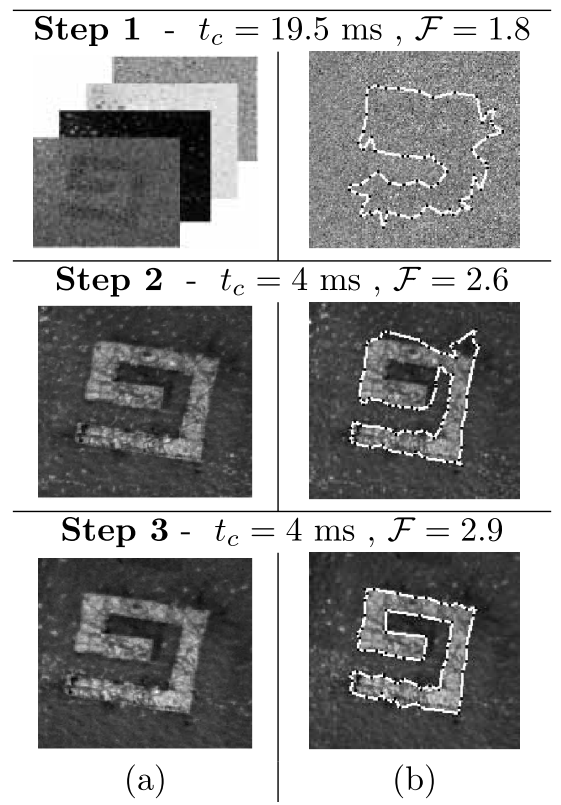

Fig. 3. (a) Data used for segmentation $(152 \times 162$ pixel images). (b) Segmentation results $(K=8) . t_{c}$ denotes computation time.

scene. We extract the polarimetric properties of the regions inside and outside this approximate contour. We check that the average Mueller matrices are physically realizable, and we compute the PSG/PSA states maximizing the contrast criterion in Eq. (4). The obtained optimal states are $\left\{\left(53^{\circ},-5^{\circ}\right),\left(36^{\circ},-5^{\circ}\right)\right\}$, where each state $(\alpha, \varepsilon)$ is defined by its azimuth $\alpha$ and its ellipticity $\varepsilon$. By implementing these polarization states on the imaging system, we acquire the image presented in Fig. 3 (Step 2). In this optimized intensity image, the contrast between the target and the background has been significantly improved. Using this image for a new 1D segmentation step, we obtain an improvement of the shape estimation. However, as some fluctuations remain in the images, the shape estimation has still some defects. The new optimal PSG/ PSA states $\left\{\left(5^{\circ},-1^{\circ}\right),\left(35^{\circ}, 7^{\circ}\right)\right\}$ estimated from this segmentation are then implemented on the imager, leading to the image in Fig. 3 (Step 3), where we observe that the contrast is sufficiently improved to yield a precise estimation of the object shape. In this case, a correct shape estimation is finally recovered in only three iterations, for a global computation time of about $30 \mathrm{~ms}$. The main benefit of this method is that each image acquisition has a duration of only $t_{0}(2.4 \mathrm{~ms}$ in the present case). A full Mueller image with sufficient SNR for each channel would require a total acquisition time of $16 t_{0}$ ( $t_{0}$ for each channel), and the scene should not evolve during this time interval. This method is thus particularly beneficial for observing rapidly evolving scene, which is a difficult problem for polarization imaging.

The improvement of separability between the estimated target and the background throughout the process can be quantified by computing the Fisher ratio [Eq. (4)] at each step of the process. For Step 1, since the image is multidimensional, the Fisher ratios obtained for each of the 16 channels are summed (this is only an approximation since it assumes that the perturbations in each image are uncorrelated), and for the subsequent steps, the Fisher ratio is directly computed on the optimized intensity image. The values of the Fisher ratio obtained at the end of the different steps are given in Fig. 3 . We can see that they constantly increase during the process.

We have presented a methodology for automatic target detection based on the iterative interplay between an active polarimetric imager with adaptive capabilities and a snake-based image segmentation algorithm. It does not require prior knowledge of the polarimetric properties of the scene and provides a scalar image with optimal contrast that can be further exploited by a human observer. These results illustrate the benefits of integrating digital processing algorithms at the heart of the image acquisition process rather than using them only for postprocessing.

Guillaume Anna's Ph.D. thesis is supported by the Délégation Générale pour l'Armement (DGA), Mission pour la Recherche et l'Innovation Scientifique (MRIS).

\section{References}

1. J. S. Tyo, M. P. Rowe, E. N. Pugh, and N. Engheta, Appl. Opt. 35, 1855 (1996).

2. J. E. Solomon, Appl. Opt. 20, 1537 (1981).

3. A. B. Kostinski and W. M. Boerner, IEEE Trans. Antennas Propag. 35, 988 (1987).

4. A. B. Kostinski, B. D. James, and W. M. Boerner, J. Opt. Soc. Am. A 5, 58 (1988).

5. A. B. Kostinski, B. D. James, and W.-M. Boerner, Can. J. Phys. 871 (1988).

6. G. Anna, F. Goudail, and D. Dolfi, Opt. Lett. 36, 4590 (2011).

7. F. Goudail and A. Bénière, Opt. Lett. 34, 1471 (2009).

8. F. Galland and P. Réfrégier, Opt. Lett. 30, 2239 (2005).

9. N. Bertaux, F. Galland, and P. Réfrégier, Electron. Lett. 47, 594 (2011). 